\title{
Horn Renamability and Hypergraphs
}

Dušan Hvalica

Faculty of Economics, University of Ljubljana, Slovenia
Satisfiability testing in the context of directed hypergraphs is discussed. A characterization of Hornrenamable formulae is given and a subclass of SAT that belongs to $\mathcal{P}$ is described. Two algorithms for Horn renaming with linear time complexity are presented.

Keywords: satisfiability, Horn, renamable, hypergraph

\section{Introduction}

The satisfiability problem, SAT, consisting of testing the satisfiability of a propositional formula, is known to be $\mathcal{N} \mathcal{P}$-complete. On the other hand, several subclasses of propositional formulae are known, so that the restriction of SAT to such a subclass is solvable in polynomial time $[7,2]$ - among them Horn formulae [5] and formulae that are reducible to Horn, such as Horn-renamable formulae $[10,4,1,3,9]$. Naturally, these classes have attracted much attention.

SAT can be translated in a natural way into the context of directed hypergraphs - testing of satisfiability translates to searching for a zerocardinality cut $[6,8]$.

The aim of this paper is to show that the hypergraph approach to SAT gives rise to new concepts, advantageous in designing algorithms and in specifying classes of formulae. We demonstrate this by giving a characterization of Horn-renamable formulae in terms of directed hypergraphs (as a by-product we obtain a subclass of SAT that belongs to $\mathcal{P}$ ); we also describe two algorithms for Horn renaming with linear time complexity.

\section{Definitions and Notation}

An (oriented) hypergraph $G$ is defined as $G=$ $(\mathcal{V}, \mathcal{A})$, where $\mathcal{V}$ and $\mathcal{A}$ are the sets of nodes and hyperarcs, respectively. A hyperarc $\mathcal{E}$ is defined as $\mathcal{E}=(T(\mathcal{E}), H(\mathcal{E}))$, where $T(\mathcal{E}), H(\mathcal{E}) \subset \mathcal{V}$; the sets $T(\mathcal{E})$ and $H(\mathcal{E})$ are called the tail and head of $\mathcal{E}$, respectively. A hyperarc, whose head has at most one element, is called a $B$-arc (backward (hyper)arc), a hypergraph, the hyperarcs of which are all B-arcs, is a B-graph.

A subhypergraph of a hypergraph $G=(\mathcal{V}, \mathcal{A})$ is a hypergraph $G_{1}=\left(\mathcal{V}_{1}, \mathcal{A}_{1}\right)$ such that $\mathcal{V}_{1} \subset$ $\mathcal{V}$ and $\mathcal{A}_{1} \subset \mathcal{A}$. When suitable, $\mathcal{V}_{1}$ will be denoted by $\mathcal{V}\left(G_{1}\right)$ and $\mathcal{A}_{1}$ by $\mathcal{A}\left(G_{1}\right)$.

For any node $u$ its backward star $\mathrm{BS}(u)$ is defined by $\mathrm{BS}(u)=\{\mathcal{E} ; u \in H(\mathcal{E})\}$, while its forward star $\operatorname{FS}(u)$ is $\operatorname{FS}(u)=\{\mathcal{E} ; u \in T(\mathcal{E})\}$. A node $u$ for which $\operatorname{BS}(u)=\emptyset$ or $\operatorname{FS}(u)=\emptyset$ will be called a tip node.

A path is a sequence $u_{1}, \mathcal{E}_{1}, u_{2}, \mathcal{E}_{2}, \ldots, \mathcal{E}_{q-1}, u_{q}$, such that $u_{i} \in H\left(\mathcal{E}_{i-1}\right)$ for $1<i \leq q$ and $u_{i} \in T\left(\mathcal{E}_{i}\right)$ for $1 \leq i<q$. If $u_{q} \in T\left(\mathcal{E}_{1}\right)$, such a path is called a cycle.

Let $S$ be any set of nodes in a $B$-graph $G$. A $B$-hyperpath or, shortly, B-path, based on $S$ and ending at $t$ is any hypergraph $P$, which is a minimal subhypergraph of $G$ such that

- $t \in \mathcal{V}(P)$,

- for every $v \in \mathcal{V}(P)$ there exists in $P$ a simple cycle-free path from some $u \in S$ to $v$.

When such a $B$-path exists, we also say that $t$ is $B$-connected to $S$. 


\section{Satisfiability of Propositional Formulae}

Let $A$ be a set of propositional variables and $B$ a set of clauses over $A$, i.e., of formulae of the form:

$$
C_{1} \wedge \cdots \wedge C_{m} \Rightarrow D_{1} \vee \cdots \vee D_{n},
$$

where $C_{1}, \ldots, C_{m}, D_{1}, \ldots, D_{n}$ belong to $A \cup$ $\{$ true, false $\}$. We say that $B$ is satisfiable if there exists a truth assignment $A \rightarrow\{$ true, false $\}$ such that every clause in $B$ is true.

The satisfiability problem, SAT, consists of testing the satisfiability of $B$. As already pointed out, it is $\mathcal{N} \mathcal{P}$-complete.

A clause (1) with $n \leq 1$ is known as a Horn clause. If every clause in $B$ is a Horn clause, we speak of Horn-SAT. It is known that it is solvable in linear time.

To every instance of SAT a hypergraph can be assigned in the following way: its nodes are the propositions, while its hyperarcs correspond to clauses such that the hyperarc corresponding to clause (1) is $\left(\left\{C_{1}, \ldots, C_{m}\right\},\left\{D_{1}, \ldots, D_{n}\right\}\right)$. If every clause is a Horn clause, the resulting hypergraph is clearly a $B$-graph. Moreover, in that case the problem of satisfiability translates into the problem of verifying $B$-connectedness in this $B$-graph; in general, a formula is satisfiable if and only if there is a 0 -cardinality cut in the corresponding hypergraph (for details, see [6]).

One of the polynomially solvable subclasses of SAT is the class of Horn-renamable formulae, i.e., $\mathrm{CNF}$ formulae, for which a renaming (replacing, for some variables $x_{i}, i \in I$, each occurrence of $x_{j}$ and $\bar{x}_{j}$ by $\bar{y}_{j}$ and $y_{j}$, respectively) exists that turns the formula into a Horn formula. Clearly, every replacement of $x_{j}$ and $\bar{x}_{j}$ by $\bar{y}_{j}$ and $y_{j}$ in the formula corresponds to a switch of position of node $x_{j}$ in every hyperarc of the corresponding hypergraph - if $x_{j} \in T(\mathcal{E})$ we move it to $H(\mathcal{E})$ and vice versa. Thus, a formula is Horn-renamable iff there exists a switching that turns the corresponding hypergraph into a $B$-graph.

\section{Hypergraphs and $B$-graphs}

Let us introduce the following new concept:
Definition 1. A track is a sequence

$$
u_{1}, \mathcal{E}_{1}, u_{2}, \mathcal{E}_{2}, \ldots, \mathcal{E}_{q-1}, u_{q}
$$

such that

- for any triple $u_{i}, \mathcal{E}_{i}, u_{i+1}$ on the track we have $u_{i} \neq u_{i+1}$ and $u_{i}, u_{i+1} \in T\left(\mathcal{E}_{i}\right) \cup H\left(\mathcal{E}_{i}\right)$,

- for any triple $\mathcal{E}_{i}, u_{i+1}, \mathcal{E}_{i+1}$ on the track we have $\mathcal{E}_{i} \neq \mathcal{E}_{i+1}$ and one of the following holds:

$$
\begin{aligned}
& -u_{i+1} \in H\left(\mathcal{E}_{i}\right) \text { and } u_{i+1} \in T\left(\mathcal{E}_{i+1}\right), \\
& -u_{i+1} \in T\left(\mathcal{E}_{i}\right) \text { and } u_{i+1} \in H\left(\mathcal{E}_{i+1}\right) .
\end{aligned}
$$

If $u_{q-1}, \mathcal{E}_{q-1}, u_{q}, \mathcal{E}_{1}, u_{2}$ is a track, then track (2) is called a hypercycle (of course, in such case either $u_{q} \in T\left(\mathcal{E}_{1}\right)$ or $u_{q} \in H\left(\mathcal{E}_{1}\right)$; often we have $u_{q}=u_{1}$ ).

Thus, a track consists of paths and "reversed" paths glued together at common hyperarcs.

For instance, $u_{1}, \mathcal{E}_{1}, u_{4}, \mathcal{E}_{2}, u_{5}, \mathcal{E}_{4}, u_{6}, \mathcal{E}_{3}, u_{4}$, $\mathcal{E}_{5}, u_{3}$ is a track in the hypergraph in Figure 1.

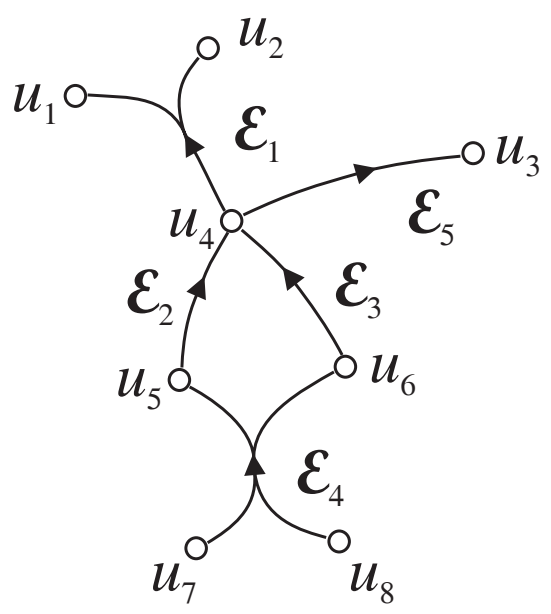

Figure 1.

If $T=u_{1}, \mathcal{E}_{1}, \ldots, \mathcal{E}_{i-1}, u_{i}$ and $T^{\prime}=u_{i}, \mathcal{E}_{1}^{\prime}, \ldots$, $\mathcal{E}_{j-1}^{\prime}, u_{j}^{\prime}$ are tracks, we shall denote

$$
T T^{\prime}=u_{1}, \mathcal{E}_{1}, \ldots, \mathcal{E}_{i-1}, u_{i}, \mathcal{E}_{1}^{\prime}, \ldots, \mathcal{E}_{j-1}^{\prime}, u_{j}^{\prime} .
$$

provided that $T T^{\prime}$ is also a track (which happens when $\mathcal{E}_{i-1}$ and $\mathcal{E}_{1}^{\prime}$ do not both belong to $\operatorname{BS}\left(u_{i}\right)$ or to $\operatorname{FS}\left(u_{i}\right)$.

Similarly, for any tracks $T=u_{1}, \mathcal{E}_{1}, \ldots, \mathcal{E}_{i-1}, u_{i}$ and $T^{\prime}=u_{i}^{\prime}, \mathcal{E}_{1}^{\prime}, \ldots, \mathcal{E}_{j-1}^{\prime}, u_{j}^{\prime}$ and hyperarc $\mathcal{E}$, we shall denote

$T \mathcal{E} T^{\prime}=u_{1}, \mathcal{E}_{1}, \ldots, \mathcal{E}_{i-1}, u_{i}, \mathcal{E}, u_{i}^{\prime}, \mathcal{E}_{1}^{\prime}, \ldots, \mathcal{E}_{j-1}^{\prime}, u_{j}^{\prime}$ 
provided that $T \mathcal{E} T^{\prime}$ is a track as well.

For any track $T=u_{1}, \mathcal{E}_{1}, \ldots, \mathcal{E}_{i-1}, u_{i}$ its reverse track is $T^{-1}=u_{i}, \mathcal{E}_{i-1}, \ldots, \mathcal{E}_{1}, u_{1}$. Clearly we have $\left(T_{1} T_{2}\right)^{-1}=T_{2}^{-1} T_{1}^{-1}$.

If tracks $T_{1}$ and $T_{2}$ have a common node $v$, we shall say that they lead in the same direction at $v$ if $T_{1}^{\prime}\left(T_{2}^{\prime}\right)^{-1}$, where $T_{1}^{\prime}$ and $T_{2}^{\prime}$ denote the subtracks of $T_{1}$ and $T_{2}$, respectively, ending at $v$, is not a track.

Any track $x, \mathcal{E}_{1}, \ldots, \mathcal{E}_{2}, x$ such that either $\mathcal{E}_{1}, \mathcal{E}_{2}$ $\in \mathrm{BS}(x)$ or $\mathcal{E}_{1}, \mathcal{E}_{2} \in \mathrm{FS}(x)$ will be called a $r e-$ turn twist. Thus, if $T$ is a return twist and $T^{\prime} T$ is a track, then $T T^{\prime-1}$ is also a track. If $T_{1}$ is a return twist and $T=T^{\prime} T_{1}$ is a track, we shall say that $T$ ends with a return twist. If $T_{1}, T_{2}$ are return twists and $T=T_{1} T^{\prime} T_{2}$ is a track, we shall say that $T$ has return twists at both ends.

A track is simple if every node and hyperarc appear only once in the sequence. A hypercycle is simple if it is a simple track or its first and last node are the only elements in the sequence that appear more than once. A return twist is simple if its first and last node and hyperarc are the only elements in the sequence that may appear more than once.

For any $t \in \mathcal{V}(G)$, by $G_{t}^{-}$(resp. $G_{t}^{+}$) we shall denote the subhypergraph, consisting of the nodes and hyperarcs lying on any track $T=$ $t, \mathcal{E}, \ldots$ such that $\mathcal{E} \in \mathrm{BS}(t)($ resp. $\mathcal{E} \in \mathrm{FS}(t))$ and that $T$ is simple or of the form $T=T_{1} T_{2}$, where $T_{1}$ is simple and $T_{2}$ is a simple return twist or hypercycle.

Consider the hypergraph in Figure 2.

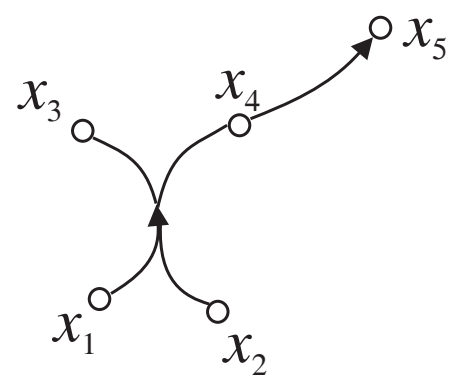

Figure 2.

Clearly, if the position of $x_{1}$ must be switched, then to get a $B$-graph the position of $x_{2}$ must not be switched, while the position of $x_{3}$ and $x_{4}$ must be switched. If the position of $x_{3}$ must not be switched, then to get a $B$-graph the position of $x_{4}$ must be switched, while the position of $x_{1}$ and $x_{2}$ must not be switched.

It follows that, if a hypergraph is to be transformed into a $B$-graph, the need that a node is (resp. is not) switched, propagates along tracks: the need to be switched propagates along subtracks in the direction of hyperarcs, while the need not to be switched propagates along subtracks in the opposite direction of hyperarcs. Hence, the following applies:

Lemma 2. If every hyperarc on the track $T=x_{1}, \mathcal{E}_{1}, \ldots, \mathcal{E}_{2}, x_{2}$ is to be turned into a $B$-arc, then if any of the following conditions is satisfied:

- $\mathcal{E}_{1} \in \mathrm{FS}\left(x_{1}\right)$ and the position of $x_{1}$ is switched,

- $\mathcal{E}_{1} \in \operatorname{BS}\left(x_{1}\right)$ and the position of $x_{1}$ is not switched,

then the following applies:

- if $\mathcal{E}_{2} \in \mathrm{BS}\left(x_{2}\right)$, then $x_{2}$ must be switched,

- if $\mathcal{E}_{2} \in \mathrm{FS}\left(x_{2}\right)$, then $x_{2}$ must not be switched.

Corollary 3. If there exists a return twist $T=x, \mathcal{E}_{1}, \ldots, \mathcal{E}_{2}, x$, then - if all hyperarcs on $T$ are to be turned into $B$-arcs — the position of $x$

- must be switched when $\mathcal{E}_{1}, \mathcal{E}_{2} \in \mathrm{BS}(x)$,

- must not be switched when $\mathcal{E}_{1}, \mathcal{E}_{2} \in \mathrm{FS}(x)$.

Proof. If $\mathcal{E}_{1}, \mathcal{E}_{2} \in B S(x)$, then if $x$ must not be switched, by Lemma 2, it must be switched. Thus it is impossible that $x$ is not switched. The other assertion can be proven analogously.

Corollary 4. If every hyperarc on the track $T=x, \mathcal{E}, \ldots$ which ends with a return twist is to be turned into a $B$-arc, then

- if $\mathcal{E} \in \mathrm{BS}(x)$, then $x$ must be switched,

- if $\mathcal{E} \in \mathrm{FS}(x)$, then $x$ must not be switched.

Proof. Let $T=T_{1} R$, where $R$ is the return twist and let $T_{1}=\ldots, \mathcal{E}^{\prime}, x^{\prime}$ and $R=$ $x^{\prime}, \mathcal{E}_{1}, \ldots, \mathcal{E}_{2}, x^{\prime}$. If $\mathcal{E}_{1}, \mathcal{E}_{2} \in B S\left(x^{\prime}\right)$, then by Corollary $4 x^{\prime}$ must be switched, so that, as then $\mathcal{E}^{\prime} \in F S\left(x^{\prime}\right)$, by Lemma 2 applied to $T_{1}^{-1}, x$ must be switched as well. If $\mathcal{E}_{1}, \mathcal{E}_{2} \in F S\left(x^{\prime}\right)$, then $x^{\prime}$ must not be switched, so that, as then $\mathcal{E}^{\prime} \in B S\left(x^{\prime}\right), x$ must be switched again. The other assertion can be proved analogously.

Suppose now that in a hypergraph $G$ there exists a track $T$ with return twists at both ends - let 
$T=R_{1} T^{\prime} R_{2}$, where $R_{1}$ and $R_{2}$ are return twists. Let $v$ be a node on $T^{\prime}$ and denote by $T_{1}$ and $T_{2}$ the subtracks of $T$ ending resp. beginning at $v$. Then $T_{1}^{-1}$ and $T_{2}$ are tracks that end with a return twist and since their first hyperarcs belong one to $\operatorname{FS}(v)$ and the other to $\mathrm{BS}(v)$, by Corollary 4 , if all hyperarcs on $T$ are to be turned into $B$-arcs, $v$ must be switched and must not be switched - which is a contradiction. Hence, if in a hypergraph there exists a track with return twists at both ends, by switching the position of its nodes such hypergraph cannot be turned into a $B$-graph.

Thus, the condition that the hypergraph does not contain tracks with return twists at both ends is a necessary condition for turning the hypergraph into a $B$-graph by switching the position of its nodes; we shall show that this condition is also sufficient.

Suppose that for some $u \in \mathcal{V}(G)$ there exists some $t \in \mathcal{V}(G)$ and tracks $T_{1}, T_{2}$ from $u$ to $t$ that do not lead in the same direction at $t$. Then, clearly, $T_{1} T_{2}^{-1}$ is a track which is a return twist.

Thus, if in $G_{u}^{+}$there are no tracks starting at $u$ and ending with a return twist, for every $t$ $\in \mathcal{V}\left(G_{u}^{+}\right)$all tracks through $t$ lead in the same direction. It follows that there is a well defined unary relation $S \subset \mathcal{V}\left(G_{u}^{+}\right): t \in S$ if and only if in $G_{u}^{+}$there exists a track $u, \ldots, \mathcal{E}, t$, where $\mathcal{E} \in \mathrm{BS}(t)$. Analogously, if in $G_{u}^{-}$there are no tracks starting at $u$ and ending with a return twist, there is a well defined unary relation $S \subset \mathcal{V}\left(G_{u}^{-}\right): t \in S$ if and only if in $G_{u}^{-}$there exists a track $u, \ldots, \mathcal{E}, t$, where $\mathcal{E} \in \operatorname{BS}(t)$.

Suppose now that in $G$ there are no tracks with return twists at both ends. Let $R=$ $x, \mathcal{E}_{1}, \ldots, \mathcal{E}_{2}, x$ be a return twist and $\mathcal{E}_{1}, \mathcal{E}_{2} \in$ $\operatorname{BS}(x)$. Then in $G_{x}^{+}$there are no tracks starting at $x$ and ending with a return twist so that there is a well defined unary relation $S \subset \mathcal{V}\left(G_{x}^{+}\right), t \in S$ if and only if in $G_{x}^{+}$there exists a $\operatorname{track} x, \ldots, \mathcal{E}, t$, where $\mathcal{E} \in \operatorname{BS}(t)$. If $G$ is to be turned into a $B$-graph, by Corollary $3, x$ must be switched, but then, by Lemma 2, every $t \in S$ must be switched. Suppose now that $x$ and every $t \in S$ are indeed switched and let $\mathcal{E}$ be any hyperarc in $G_{x}^{+}$, let moreover $T=x, \ldots, \mathcal{E}^{\prime}, v^{\prime}, \mathcal{E}, v$ be a track such that besides $v$ and $v^{\prime}$ no other node in $H(\mathcal{E}) \cup T(\mathcal{E})$ lies on $T$. If $v^{\prime} \in T(\mathcal{E})$, then $v^{\prime} \in H\left(\mathcal{E}^{\prime}\right)$ so that $v^{\prime} \in S$. Of course, for every $v \in T(\mathcal{E}) \backslash\left\{v^{\prime}\right\}$ we have $v \notin S$ and for every $v \in H(\mathcal{E})$ we have $v \in S$. Thus, after the switch, we have $H(\mathcal{E})=\left\{v^{\prime}\right\}$, so that $\mathcal{E}$ becomes a $B$-arc. If $v^{\prime} \in H(\mathcal{E})$, then $v^{\prime} \in T\left(\mathcal{E}^{\prime}\right)$ so that $v^{\prime} \notin S$ and $v \notin S$ for every $v \in T(\mathcal{E})$ and $v \in S$ for every $v \in H(\mathcal{E}) \backslash\left\{v^{\prime}\right\}$, so that after the switch we also have $H(\mathcal{E})=\left\{v^{\prime}\right\}$ and $\mathcal{E}$ becomes a $B$-arc again.

Similarly, if $R=x, \mathcal{E}_{1}, \ldots, \mathcal{E}_{2}, x$ is a return twist and $\mathcal{E}_{1}, \mathcal{E}_{2} \in \mathrm{FS}(x)$, there is a well defined unary relation $S \subset \mathcal{V}\left(G_{x}^{-}\right), t \in S$ if and only if in $G_{x}^{-}$ there exists a track $x, \ldots, \mathcal{E}, t$, where $\mathcal{E} \in \operatorname{BS}(t)$, and if the position of the nodes in $S$ is switched, every hyperarc in $G_{x}^{-}$is turned into a $B$-arc. For any return twist $R=x, \ldots, x$ we shall denote by $G_{x}^{ \pm}$the appropriate subhypergraph of the pair $G_{x}^{+}, G_{x}^{-}$(i.e., such that if $T=x, \ldots$ is a track in $G_{x}^{ \pm}$then $R T$ is also a track).

When for return twists $R_{1}=x_{1}, \ldots, x_{1}$ and $R_{2}=x_{2}, \ldots, x_{2}$ hypergraphs $G_{x_{1}}^{ \pm}$and $G_{x_{2}}^{ \pm}$overlap, relations $S_{x_{1}}$ and $S_{x_{2}}$ agree on the intersection: if there exist tracks $T_{1}=x_{1}, \ldots, t$ in $G_{x_{1}}^{ \pm}$ and $T_{2}=x_{2}, \ldots, t$ in $G_{x_{2}}^{ \pm}$, they lead in the same direction at $t$ (otherwise $T_{1} T_{2}^{-1}$ would be a track and $R_{1} T_{1} T_{2}^{-1} R_{2}$ a track with return twists at both ends). Thus, relation $S$ is defined on $\sum_{x \in R} G_{x}^{ \pm}$, where $R$ denotes the set of all nodes which are the starting nodes of a return twist in $G$.

Suppose now that $\mathcal{V}\left(\sum_{x \in R} G_{x}^{ \pm}\right) \neq \mathcal{V}(G)$ and take any $t \in \mathcal{V}(G) \backslash \mathcal{V}\left(\sum_{x \in R} G_{x}^{ \pm}\right)$. Since, for any $x \in R$, in $G_{x}^{ \pm}$there is no track from $x$ to $t$, in $G_{t}^{+}$there are no tracks starting at $t$ and ending with a return twist, so that $S$ is well defined on $G_{t}^{+}$. Moreover, for any $x \in R$, it agrees with $S$ defined on $G_{x}^{ \pm}\left(\right.$any tracks $T_{1}=x, \ldots, v$ in $G_{x}^{ \pm}$ and $T_{2}=t, \ldots, v$ in $G_{t}^{+}$lead in the same direction at $v$, for otherwise $T_{1} T_{2}^{-1}$ would be a track from $x$ to $t$, but by our assumption, $\left.t \notin \mathcal{V}\left(G_{x}^{ \pm}\right)\right)$. If $\mathcal{V}\left(G_{t}^{+}+\sum_{x \in R} G_{x}^{ \pm}\right) \neq \mathcal{V}(G)$, the argument can be repeated, until finally $\mathcal{V}\left(\sum_{x} G_{x}^{ \pm}\right)=$ $\mathcal{V}(G)$ so that $S$ is well defined on $G$. We know that when the position of the nodes in $S$ is switched, every hyperarc in $\mathcal{A}\left(\sum_{x} G_{x}^{ \pm}\right)$turns into a $B$-arc. But this applies to other hyperarcs (if any) as well: if $\mathcal{E} \in \mathcal{A}(G) \backslash \mathcal{A}\left(\sum_{x} G_{x}^{ \pm}\right)$, then we must have $H(\mathcal{E}) \subset S$ and $T(\mathcal{E}) \cap S=\emptyset$ (for any $v \in H(\mathcal{E})$ there exists in some $G_{x}^{ \pm}$a track $T=x, \ldots, \mathcal{E}_{1}, v$ and since $\mathcal{E}_{1} \in \mathrm{FS}(v)$ would imply $\mathcal{E} \in \mathcal{A}\left(G_{x}^{ \pm}\right)$, we must have $\mathcal{E}_{1} \in \mathrm{BS}(v)$ and therefore $v \in S$; similarly one verifies that 
$v \notin S$ for any $v \in T(\mathcal{E}))$. Thus, after the switch $H(\mathcal{E})=\emptyset$ so that $\mathcal{E}$ is a $B$-arc.

It follows that when in a hypergraph $G$ there are no tracks $T$ with return twists at both ends, then in $\mathcal{V}(G)$ there exists a unary relation $S$ such that when the position of the nodes in $S$ is switched, every hyperarc in $G$ turns into a $B$-arc.

We have proved:

Theorem 5. A hypergraph can be turned into a $B$-graph by switching the position of nodes if and only if it does not contain any tracks with return twists at both ends.

For instance, the hypergraph in Figure 3 cannot be turned into a $B$-graph, as for $T_{1}=$ $x_{3}, \mathcal{E}_{3}, x_{6}, \mathcal{E}_{5}, x_{7}, \mathcal{E}_{4}, x_{3}, T_{2}=x_{5}, \mathcal{E}_{7}, x_{12}, \mathcal{E}_{6}, x_{13}$, $\mathcal{E}_{8}, x_{15}, \mathcal{E}_{9}, x_{14}, \mathcal{E}_{7}, x_{5}$ and $T^{\prime}=x_{3}, \mathcal{E}_{1}, x_{4}, \mathcal{E}_{2}, x_{5}$, the track $T=T_{1} T^{\prime} T_{2}$ is a track with return twists at both ends.

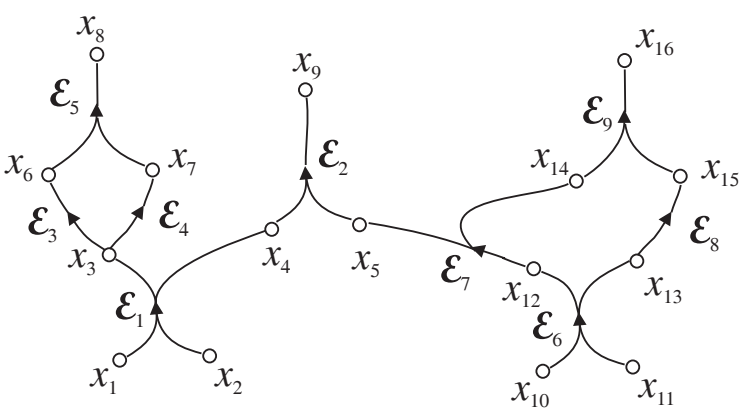

Figure 3.

Clearly, if $T=T_{1} T^{\prime} T_{2}$ is a track with return twists at both ends, then $T_{1} T^{\prime} T_{2} T^{\prime-1}$ is a hypercycle. Conversely, suppose that $T$ is a hypercyle which has a subtrack $T^{\prime}$ that is a return twist. Denote $T=T_{1} T^{\prime} T_{2}$, then, clearly, $T_{2} T_{1}$ is also a return twist and $T^{\prime} T_{2} T_{1}$ is a track with return twist at both ends.

Hence, we have the following

Lemma 6. A hypergraph contains tracks with return twists at both ends if and only if it contains hypercycles with a subtrack that is a return twist.

Now we can apply our results to the issue of satisfiability.

Theorem 7. A propositional formula is Hornrenamable if and only if the corresponding hypergraph does not contain any tracks with return twists at both ends.
Corollary 8. A propositional formula is Hornrenamable if and only if the corresponding hypergraph does not contain any hypercycles with a subtrack that is a return twist.

Corollary 9. A propositional formula for which the corresponding hypergraph does not contain any hypercycles is Horn-renamable.

The class of propositional formulae for which the corresponding hypergraph is without hypercycles is therefore a proper subclass of the class of Horn-renamable formulae. Thus, we have found a new class such that the restriction of SAT to it is solvable in polynomial time. Of course, this class being a subclass of Hornrenamable formulae, our result is not of much importance as far as practical algorithms are concerned. Still, being a different subclass it may have some relevance in the tractability versus intractability issue. Moreover, it can be shown that the corresponding hypergraphs have some nice properties.

\section{Horn-renaming}

Testing for Horn renamability can be based on Corollary 8. Tracks are extended in a depthfirst manner until the track ends at a tip node or it joins a previously constructed track or a hypercycle is completed. If a return twist is completed, a flag is raised for the time of extending that track and if a hypercycle is completed when the flag is raised the algorithm stops with the output ' $a$ hypercycle containing $a$ return twist'. Clearly each node can be passed through only once in each direction so that the time complexity of the algorithm is $O(|G|)$, where $|G|=\sum_{\mathcal{E} \in \mathcal{A}}(|H(\mathcal{E})|+|T(\mathcal{E})|$ ) (which is comparable to the time complexity of the algorithms from $[3,9])$.

As already mentioned, solving instances of HornSAT can be done in the context of $B$-graphs, by searching for a $B$-path. Thus, to practically solve an instance of (general) SAT in the context of hypergraphs, one can start by attempting to turn the given hypergraph into a $B$-graph, i.e., by searching for a set $S$ of the nodes which are to be switched to turn the hypergraph in question into a $B$-graph. 
An algorithm to this end can be based on Lemma 2 and Corollary 4, specifically, it can be designed as follows: it starts by tentatively labelling (while extending tracks in a depth-first manner) the nodes of the hypergraph according to Lemma 2 - label 'to be switched' is propagated in the direction of hyperarcs, while label 'not to be switched' is propagated in the opposite direction. This can proceed until a return twist is found; when this occurs, final labels are set (again while extending tracks in a depth-first manner) according to Corollary 4 - label 'must be switched' is propagated along the hyperarcs while label 'must not be switched' is propagated in the opposite direction. If another return twist is found in this phase the algorithm stops with the output 'a track with return twists at both ends', otherwise the set of the nodes labelled 'must be switched' or 'to be switched' is the desired $S$. As the label of any node can change only twice (when a tentative label is replaced by a fixed one), the propagation of labels through any node can occur only twice. Consequently, the time complexity of this algorithm is also $O(|G|)$. This algorithm is quite similar to the algorithm RHSat in [11], except that, of course, it operates in the corresponing hypergraph.

\section{Conclusion}

We have introduced the concept of a track and, based on it, found a necessary and sufficient condition that a hypergraph can be turned into a $B$-graph. This yields a characterization of Horn-renamable formulae. Furthermore, we have found a new class of formulae such that the restriction of SAT to it is solvable in polynomial time.

We also present two algorithms for testing whether a given hypergraph can be turned into a $B$-graph and (if so) for performing this change. As these algorithms have linear time complexity (which is comparable to the best known results), it seems that the hypergraph approach to SAT is worthy of further study.

\section{References}

[1] E. Boros, P. L. HAMMER, X. Sun, Recognition of q-Horn formulae in linear time. Discrete Applied Mathematics, 55, 1-13, 1994.

[2] O. CEPEK, P. KUCERA, Known and new classes of generalized Horn formulae with polynomial recognition and SAT testing. Discrete Applied Mathematics, 149, 14-52, 2005.

[3] V. Chandru, C. R. Coullard, P. L. Hammer, M. MonTANEZ, X. SUN, On renamable Horn and generalized Horn functions. Annals of Math. and Art. Intel., 1, 33-47, 1990.

[4] V. Chandru, J. N. HoOKer, Extended Horn sets in propositional logic. Journal of the Association for Computing Machinery, 38, 205-221, 1991.

[5] W. F. Dowling, J. H. GALLIER, Linear time algorithms for testing the satisfiability of Horn formulae. J. Logic Programming, 1, 207-284, 1984.

[6] G. Gallo, G. Longo, S. Pallottino, S. Nguyen, Directed Hypergraphs and Applications. Discrete Applied Mathematics, 42, 177-201, 1993.

[7] G. Gallo, M. G. Scutella, Polynomially solvable satisfiability problems. Information Processing Letters, 29, 221-227, 1988.

[8] G. Gallo, C. Gentile, D. Pretolani, G. Rago, Max Horn SAT and the Minimum Cut Problem in Directed Hypergraphs. Mathematical Programming, 80, 213-237, 1998.

[9] J. J. HEBRARD, A linear algorithm for renaming a set of clauses as a Horn set. Theoretical Computer Science, 124, 343-350, 1994.

[10] H. R. LEWIS, Renaming a set of clauses as a Horn set. Journal of the ACM, 25, 134-135, 1978.

[11] A. DEL VAL, On 2-SAT and Renamable Horn. Proceedings of AAAI/IAAI 2000, 279-284, 2000.

Received: June, 2008 Accepted: February, 2009

Contact address:

Dušan Hvalica Faculty of Economics University of Ljubljana Kardeljeva ploščad 17 $\mathrm{Si}-1000$ Ljubljana Slovenia e-mail: dusan.hvalica@ef.uni-lj.si

Dušan Hvalica received his Ph. D. in mathematics in 1985. He is an Associate professor at the faculty of Economics, University of Ljubljana. 